\title{
Does the Presence or Absence of DESH Predict Outcomes in Adult Hydrocephalus?
}

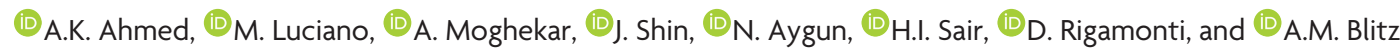

\begin{abstract}
BACKGROUND AND PURPOSE: The DESH (disproportionately enlarged subarachnoid-space hydrocephalus) pattern of "tight highconvexity and medial subarachnoid spaces, and enlarged Sylvian fissures with ventriculomegaly" is used to determine which patients undergo an operation for adult hydrocephalus at many centers. Our aim was to review adult hydrocephalus cases when DESH has not been a criterion for an operation to determine the prevalence of DESH among the cohort and compare the surgical outcomes in the presence or absence of DESH.
\end{abstract}

MATERIALS AND METHODS: A retrospective cohort study was conducted at a single institution (Johns Hopkins Hospital) to include patients surgically treated for adult hydrocephalus between 2003 and 2014 drawn from a data base of patients who had undergone standardized hydrocephalus protocol MR imaging. Preoperative imaging was reviewed by 2 blinded neuroradiologists to characterize the presence of DESH. Preoperative and postoperative clinical symptomatology was recorded. Frequencies were compared using the Fisher exact test, and nonparametric means were compared using the Mann-Whitney $U$ Test.

RESULTS: One hundred thirty-three subjects were identified and included (96 DESH absent, 37 DESH present). Shunting led to significant improvement in gait and urinary and cognitive symptoms for the overall cohort and for patients with and without DESH ( $P<.05)$. The Fisher exact test did not demonstrate any significant differences in either gait or urinary or cognitive symptom improvement between patients with or without DESH $(P>.05)$.

CONCLUSIONS: The current study demonstrated symptom improvement in patients with adult hydrocephalus following shunting, with no significant differences between subjects with and without DESH. Thus, shunt insertion for patients with adult hydrocephalus should not rely solely on the presence of preoperative DESH findings.

ABBREVIATIONS: DESH = disproportionately enlarged subarachnoid-space hydrocephalus; iNPH = idiopathic normal pressure hydrocephalus; MMSE = MiniMental State Examination; NPH = normal pressure hydrocephalus; TUG = Timed Up and Go test

A dult hydrocephalus, the communicating form known as normal pressure hydrocephalus (NPH) in the elderly population, is associated with the classic triad of gait, cognitive, and urinary disturbances. ${ }^{1,2}$ Shunting is the mainstay of treatment, but the accurate neuroradiologic diagnosis of shunt-responsive adult hydrocephalus may be difficult. As described by the NPH guidelines study group, there is no single standard for the imaging evaluation of idiopathic NPH (iNPH). ${ }^{3-5}$

Received April 17, 2018; accepted after revision August 10.

From the Departments of Neurosurgery (A.K.A., M.L., D.R.), Neurology (A.M.), and Radiology and Radiological Sciences (N.A., H.I.S., A.M.B.), Division of Neuroradiology, Johns Hopkins University School of Medicine, Baltimore, Maryland; and Department of Radiology (J.S.), University of California, San Francisco, School of Medicine, San Francisco, California.

Please address correspondence to Ari M. Blitz, MD, Division of Neuroradiology, Johns Hopkins Hospital, 600 North Wolfe St, Phipps B-126 Baltimore, MD 21287; e-mail: Ablitz1@jhmi.edu

http://dx.doi.org/10.3174/ajnr.A5820
The Study of Idiopathic Normal Pressure Hydrocephalus on Neurologic Improvement (SINPHONI) developed MR imagingbased diagnostic criteria for adult hydrocephalus, termed "disproportionately enlarged subarachnoid-space hydrocephalus" $(\mathrm{DESH}){ }^{2} \mathrm{DESH}$ is defined as "tight high-convexity and medial subarachnoid spaces, and enlarged Sylvian fissures with ventriculomegaly." Hashimoto et $\mathrm{al}^{2}$ reported that the DESH criteria, on coronal MR imaging, are a defining characteristic for iNPH. The DESH pattern is used to aid in the surgical selection of patients in some centers and is given particular weight in some geographic areas. ${ }^{6}$

The aim of the present study was to review adult hydrocephalus cases at a single institution (Johns Hopkins Hospital), where the presence or absence of DESH has not been used as a surgical criterion, to determine the prevalence of DESH among the cohort, characterize adult hydrocephalus cases by the presence of DESH criteria, and compare the outcomes of patients with and without DESH following shunting treatment. 


\section{MATERIALS AND METHODS}

\section{Study Design and Inclusion Criteria}

A retrospective chart review was conducted, following institutional review board approval (NA_00067508), which included patients who underwent treatment for adult hydrocephalus at a single institution from 2003 to 2014 selected from a data base of patients who have undergone a standardized hydrocephalus protocol clinical high-resolution MR imaging, including sagittal MPRAGE (TR, 2300 ms; TE, 1.88 ms; inversion recovery, 900; 144 partitions; 0.9-mm isotropic voxels). Patients undergoing an operation had a preoperative Evans index of $>0.3$ and symptoms of potential hydrocephalus. The inclusion criteria for screening consisted of patients $18-80$ years if age with symptomatic and radiographically confirmed adult hydrocephalus treated by surgical shunting, with sufficient preoperative imaging and a detailed medical record describing preoperative and postoperative symptoms. All included patients underwent high-resolution imaging through the hydrocephalus center for evaluation of potential adult hydrocephalus.

\section{Hydrocephalus Classification}

All preoperative images were de-identified and reviewed by 2 neuroradiologists blinded to clinical features, with discrepancies resolved through consensus, and scored for the presence or absence of all DESH criteria, based on the previously published criteria. ${ }^{4}$ However, previous reports did not include cutoff measurements for Sylvian fissure enlargement according to the DESH criteria. Consistent with the literature definition of DESH, the presence of Sylvian fissure enlargement was a qualitative observation made after thorough review of the relevant literature. The authors chose not to classify Sylvian fissure enlargement by measurement, to avoid inconsistencies with previous reports describing the DESH classification. $^{3-5}$ In an effort to include a pure sample of DESH cases and controls negative for DESH, we excluded patients who had partial features of DESH without fulfilling all criteria from this study. Excluded patients were those with either tight highconvexity and medial subarachnoid spaces without enlarged Sylvian fissures or patients with enlarged Sylvian fissures in the absence of tight high-convexity and medial subarachnoid spaces.

\section{Recorded Data}

Following retrospective review of medical records for adult patients surgically treated for hydrocephalus, demographic (age at treatment and sex) data, symptomatology, and treatment were recorded. Imaging, gait, urinary dysfunction, dementia, MiniMental State Examination (MMSE) scores, Timed Up and Go test (TUG) scores, ${ }^{7}$ Tinetti scores, and depression status were recorded preoperatively and postoperatively. Baseline gait was recorded on a scale of $0-4$ : 0 , no gait abnormality; 1 , minor gait abnormality on uneven surfaces or with regard to pace; 2 , recent history of falls; 3, requiring cane or walker assistance; and 4 , wheelchair-bound. Urinary function was recorded as the following: 0 , no urinary dysfunction; 1 , urinary urgency, hesitancy, ambiguous dysfunction, or noted by others; 2 , rare urinary dysfunction; 3, frequent urinary dysfunction; and 4, incontinent. Cognitive symptoms were recorded as the following: 0 , no cognitive symptoms; 1 , infrequent forgetfulness or ambiguous cogni- tive symptoms; 2 , mild cognitive symptoms; 3 , frequent cognitive symptoms; and 4, severe cognitive impairment in activities of daily living.

Because many studies of adult hydrocephalus suggest 1 year of follow-up, ${ }^{2-8}$ postoperative clinical outcomes were recorded close to the 1-year postoperative follow-up visit and at the last known clinical follow-up. Postoperative gait, and urinary and cognitive symptoms were graded by 3 criteria: 1 , worsening of symptoms; 2 , no improvement (ie, same as baseline); or 3, improvement in symptoms at the last known clinical follow-up.

\section{Statistical Analysis}

Frequencies were compared using the Fisher exact test, and nonparametric means were compared using the Mann-Whitney $U$ test. A $P$ value $<.05$ was considered significant. Analysis was performed by using STATA SE 12 (StataCorp, College Station, Texas).

\section{RESULTS}

\section{Patient Demographics and Baseline Characteristics}

Patients surgically treated for adult hydrocephalus during an 11year period (2003-2014) were included. Following blinded neuroradiology review of preoperative imaging for 162 adult patients surgically treated for hydrocephalus, 29 patients had partial features of DESH without fulfilling the complete DESH criteria and were excluded from this study. Of the remaining 133, ninety-six (72.2\%) did not meet any DESH criteria and 37 (27.8\%) had all DESH features present (Figure and Table 1). Of the 133 patients included in this study, $68.4 \%$ were men. The mean age at shunting was 71.7 years (range, $53-80$ years) (Table 1 ).

\section{Clinical Presentation}

Preoperatively, 14 (10.5\%) patients presented with a normal gait, $18(13.5 \%)$ presented with gait abnormalities on an uneven surface or slow pace, $54(40.6 \%)$ had a history of falls, $39(29.3 \%)$ required an assisted gait, and $8(6 \%)$ were wheelchair-bound. Of the 133 patients, those with preoperative findings of DESH ( $n=$ 37) had greater preoperative gait dysfunction compared with those without DESH $(n=96)(P<.05)$. Of those with preoperative DESH findings, only $2.7 \%$ had a normal gait compared with $13.5 \%$ of those without DESH (Table 2).

Of the cohort of 131 patients in whom urinary function was known, 43 (32.8\%) had no preoperative urinary dysfunction; 18 (13.7\%) had urgency, anxiety, or ambiguous symptoms; 43 $(32.8 \%)$ had rare or intermittent symptoms; 16 (12.2\%) had urinary dysfunction often; and $10(7.6 \%)$ had urinary incontinence at presentation. There were no significant differences between patients with or without DESH, regarding preoperative urinary dysfunction $(P>.05)$.

Similar to urinary dysfunction, there were no significant differences between patients with and without DESH with respect to preoperative dementia. In both groups, $15.8 \%$ had no dementia, preoperatively $(P>.05)$ (Table 2$)$.

The mean score for the MMSE was 26.5 (95\% CI, 7-30); for the Tinetti, it was 21.4 (95\% CI, 6-28); and for the TUG, it was 19.07 (95\% CI, 4.5-82); 55.3\% of patients presented with depression and $56.4 \%$ presented with headaches. MMSE, Tinetti, TUG, 


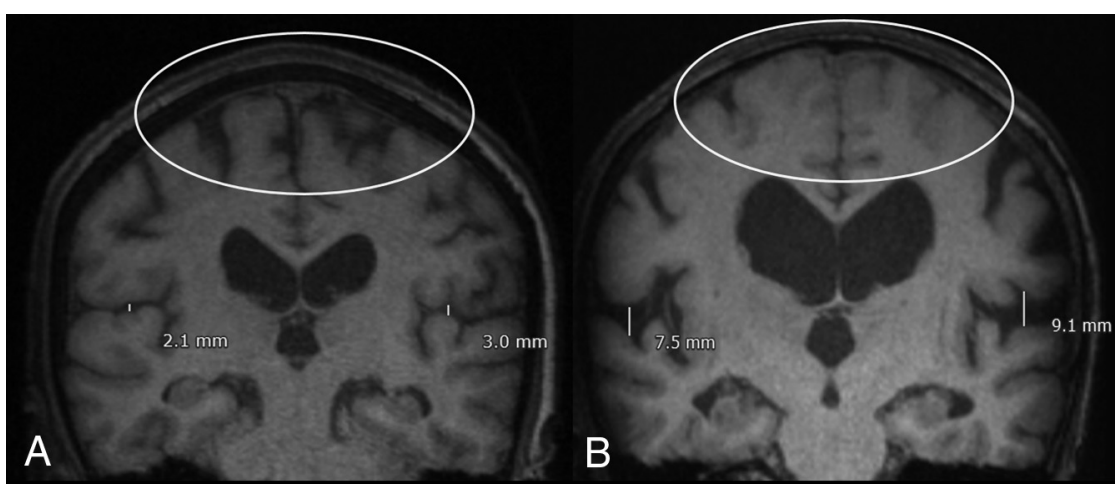

FIGURE. Characteristic imaging and classification for patients with adult hydrocephalus. $A, A b-$ sence of DESH. B, DESH present: Sylvian fissure enlargement and tight high-convexity effacement are present. Both patients were treated with ventricular shunting and had improvement in symptoms. A scaled measurement of the Sylvian fissure is provided to illustrate the extent of fissure enlargement in this representative case. However, consistent with the defining features of DESH in the literature, ${ }^{3-5}$ quantitative measurement was not used as scoring criterion during blinded neuroradiologist review.

Table 1: Demographics and baseline characteristics

\begin{tabular}{lc}
\hline \multicolumn{1}{c}{ Demographics and Characteristics } & \\
\hline Mean age (range) (yr) & $71.7(53-80)$ \\
Sex & $68.4 \%$ Male, 32.6\% female \\
Hydrocephalus classification ( $n=133)$ & \\
No DESH (No.) (\%) & $96(72.2 \%)$ \\
DESH (No.) (\%) & $37(27.8 \%)$ \\
\hline
\end{tabular}

depression, and headache status were not significant between patients with and without DESH $(P>.05$ for all) (Table 2).

\section{Clinical Outcomes following Treatment}

Clinical outcomes were recorded close to the 1-year follow-up visit $(254.3 \pm 37.9$ days $)$ and at the last known follow-up visit (1248.3 \pm 103.3 days) after the operation. There were no significant differences in any of the clinical outcomes recorded between these postoperative time points $(P>.05$ for all). Gait status was known for 113 patients following shunting treatment. Excluding those with a normal gait at baseline $(n=7), 84 \%(89 / 106)$ demonstrated gait improvement of some kind following shunting. For patients with and without preoperative DESH with preoperative gait deficits, $80 \%$ (24/30) and 85.5\% (65/76) had some magnitude of improvement in gait following shunting, respectively. Although shunting led to significant gait improvement for the overall cohort, for patients with and without DESH $(P<.05$ for all), the Fisher exact test did not demonstrate any significant differences in the magnitude or proportion of gait improvement based on the presence or absence of DESH $(P>.05)$.

Following shunting treatment, $89.9 \%$ (71/79) of patients with preoperative urinary dysfunction and $75 \%$ (30/40) with preoperative dementia symptoms had some magnitude of improvement following shunting. Shunting led to significant improvement in urinary and dementia symptoms for the overall cohort and for patients with and without DESH $(P<.05)$. The Fisher exact test did not demonstrate any significant differences in the proportion of either urinary or dementia symptom improvement between patients with or without DESH $(P>.05)$ (Table 3). The Fisher exact test also did not demonstrate any demographically significant differences with respect to sex or age for patients with known baseline and postoperative gait, urinary, and dementia symptoms $(P>.05$ for all).

The number of patients with known postoperative status for the MMSE, Tinetti, and TUG was limited and therefore not found to be statistically significant compared with preoperative values.

\section{DISCUSSION}

The communicating form of adult hydrocephalus in the elderly, NPH, is associated with the classic triad of gait, cognitive, and urinary disturbances. A third of NPH cases are classified as idiopathic, not secondary to trauma, surgery, meningitis, or other abnormalities of the arachnoid mater. ${ }^{8,9}$ Ventricular shunting may provide symptom relief in cases of NPH, which can be difficult to distinguish from vascular dementias and neurodegenerative diseases of the elderly. Thus, the treatment of NPH relies partly on accurate neuroimaging diagnosis and characterization. ${ }^{10,11}$

Kitagaki et $\mathrm{al}^{9}$ demonstrated that the Sylvian fissure CSF volume and ventricular volume were greater in patients with iNPH compared with other dementias (ie, Alzheimer disease). Patients with iNPH also had decreased CSF in the superior convexity and medial subarachnoid spaces. This study supported enlarged basal cisterns and Sylvian fissures, with focally dilated sulci, as responsive to shunting. Narita et $\mathrm{al}^{12}$ showed that presurgical high-convexity tightness, callosal angle, and Sylvian fissure dilation were significantly associated with clinical improvement. Following multiple linear regression, high-convexity tightness alone predicted clinical improvement at 1 year. However, $92 \%$ of patients had high-convexity tightness (rated as $\geq 2$ ), and $100 \%$ had Sylvian fissure dilation (rated as $\geq 2$ ). Similar to findings in the current study, findings in patients with DESH did improve following shunting, but the study of Narita et al ${ }^{12}$ excluded patients with no DESH findings who were treated by shunting.

In a study by Mostafa et al, ${ }^{13} 72$ patients negative for DESH and 31 patients positive for DESH with adult hydrocephalus were assessed for shunt responsiveness-compared with 96 and 37 patients in the current study, respectively. In this study of the 103 patients included, 78 demonstrated symptomatic improvement at 1 year after shunting. Most interesting, of those who improved, $69 \%$ were negative for DESH findings and only $31 \%$ were positive for DESH with significance between both groups $(P<.001)$. The positive and negative predictive values for DESH findings predicting shunt responsiveness were $77 \%$ and $25 \%$, respectively. This finding suggests that though the presence of DESH may predict shunt responsiveness, its absence does not preclude symptom improvement following shunting. The authors did not directly compare surgical outcomes of patients positive and negative for DESH, leaving open the possibility that DESH features were significant contributors to prognosis.

In the current study, patients positive for DESH had improvement in urinary, gait, and dementia symptoms following shunt 


\begin{tabular}{|c|c|c|c|c|}
\hline & Overall & No DESH & DESH & $\begin{array}{c}P \text { Value } \\
\text { (No DESH vs DESH) }\end{array}$ \\
\hline Gait (No.) & 133 & 96 & 37 & \\
\hline Normal gait & 14 & 13 & 1 & $>.05$ \\
\hline Gait abnormality with uneven surfaces or pace & 18 & 15 & 3 & $>.05$ \\
\hline History of falls & 54 & 40 & 14 & $>.05$ \\
\hline Assisted gait (ie, walker) & 39 & 23 & 16 & $>.05$ \\
\hline Wheelchair-bound & 8 & 5 & 3 & $>.05$ \\
\hline Urinary dysfunction (No.) & 131 & 94 & 37 & \\
\hline No dysfunction & 43 & 31 & 12 & $>.05$ \\
\hline $\begin{array}{l}\text { Urgency, anxiety, or ambiguous symptoms not } \\
\text { otherwise classified }\end{array}$ & 19 & 16 & 3 & $>.05$ \\
\hline Rare or intermittent symptoms & 43 & 30 & 13 & $>.05$ \\
\hline Urinary symptoms often & 16 & 11 & 5 & $>.05$ \\
\hline Urinary incontinence (requiring adult diapers) & 10 & 6 & 4 & $>.05$ \\
\hline Dementia (No.) & 133 & 96 & 37 & \\
\hline No dementia & 22 & 16 & 6 & $>.05$ \\
\hline Forgetfulness & 33 & 28 & 5 & $>.05$ \\
\hline Mild dementia & 47 & 33 & 14 & $>.05$ \\
\hline Moderate dementia & 21 & 14 & 7 & $>.05$ \\
\hline Severe dementia & 10 & 5 & 5 & $>.05$ \\
\hline $\mathrm{MMSE}^{\mathrm{a}}$ (mean) $(95 \% \mathrm{CI})$ & $26.5(7-30)$ & $27.1(16-30)$ & $21.3(7-28)$ & $>.05$ \\
\hline Tinetti (proportion) & $21.4(6-28)$ & $21.63(6-28)$ & $21.3(7-28)$ & $>.05$ \\
\hline TUG (mean) (95\% CI) & $19.07(4.5-82)$ & $18.63(4.5-60)$ & $19.5(9.7-82)$ & $>.05$ \\
\hline Depression (proportion) & $52 / 94(55.3 \%)$ & $40 / 73(54.8 \%)$ & $12 / 21(57.1 \%)$ & $>.05$ \\
\hline Headache (proportion) & $44 / 78(56.4 \%)$ & $36 / 62(58.1 \%)$ & $8 / 16(50 \%)$ & $>.05$ \\
\hline
\end{tabular}

${ }^{a}$ The MMSE score was available for 26/96 patients with preoperative findings without DESH and 34/37 patients with DESH.

Table 3: Outcomes following treatment for adult hydrocephalus at last known follow-up (1248.3 \pm 103.3 days following surgery) ${ }^{a}$

\begin{tabular}{|c|c|c|c|c|}
\hline & Overall & No DESH & DESH & $\begin{array}{c}P \text { Value } \\
\text { (No DESH vs DESH) }\end{array}$ \\
\hline Gait (No.) & 113 & 84 & 29 & \\
\hline Improvement & 89 & 65 & 24 & $>.05$ \\
\hline No improvement & 13 & 8 & 5 & $>.05$ \\
\hline Worse & 4 & 3 & 1 & $>.05$ \\
\hline Normal findings at baseline & 7 & 6 & 1 & $>.05$ \\
\hline$P$ value ${ }^{b}$ & $<.05$ & $<.05$ & $<.05$ & \\
\hline \multicolumn{5}{|c|}{ Improvement compared with baseline } \\
\hline Urinary dysfunction (No.) (\%) & 119 & 84 & 35 & \\
\hline Improvement & 71 & 50 & 21 & $>.05$ \\
\hline No improvement & 6 & 3 & 3 & $>.05$ \\
\hline Worse & 2 & 2 & 0 & $>.05$ \\
\hline Normal findings at baseline & 40 & 29 & 11 & $>.05$ \\
\hline$P$ value $^{\mathrm{b}}$ & $<.05$ & $<.05$ & $<.05$ & \\
\hline \multicolumn{5}{|c|}{ Improvement compared with baseline } \\
\hline \multicolumn{5}{|c|}{ Dementia (No.) (\%) } \\
\hline Improvement & 30 & 24 & 6 & $>.05$ \\
\hline No improvement & 7 & 4 & 3 & $>.05$ \\
\hline Worse & 3 & 3 & 0 & $>.05$ \\
\hline Normal findings at baseline & 7 & 4 & 3 & $>.05$ \\
\hline$P$ value $^{\mathrm{b}}$ & $<.05$ & $<.05$ & $<.05$ & \\
\hline \multicolumn{5}{|c|}{ Improvement compared with baseline } \\
\hline MMSE & $27.5(17-30)$ & $27.7(16-30)$ & $27.4(17-30)$ & $>.05$ \\
\hline Tinetti & $25(8-28)$ & $24.9(5-28)$ & $25(15-28)$ & $>.05$ \\
\hline TUG & $13.5(6-25.7)$ & $14.6(7.5-57)$ & 12.9 (10.9-19) & $>.05$ \\
\hline
\end{tabular}

${ }^{a}$ Columns compare the proportion improved among a cohort (ie, overall, DESH, No DESH); rows compare proportional improvement between those with and without DESH.

b The Fisher exact test was performed for those with preoperative symptoms, excluding those with normal findings at baseline. Findings are significant.

treatment. However, the data also demonstrated shunt responsiveness in the absence of DESH findings. Unlike previous literature, in our study, gait, dementia, and urinary symptoms improved in groups with DESH and without DESH following shunting, with no significant differences between groups. The DESH findings of tight high-convexity and medial subarachnoid spaces and enlarged Sylvian fissures with ventriculomegaly ${ }^{2}$ may therefore encompass only a portion of shunt-responsive adult hydrocephalus.

Limitations of this work include a sample size from a single institution, and those inherent in retrospective studies. In particular, outcome data available in the medical record for subjects in the study were limited and not homogeneous among all subjects. Because patients who fulfilled only partial features of DESH were 
excluded from this study, the findings presented cannot be generalized to all patients with adult hydrocephalus. The exclusion of patients with poor clinical documentation or insufficient follow-up is unavoidable when collecting meaningful data but introduces the potential for bias in the inclusion of subjects. Our data suggest that if there is prognostic significance for the DESH pattern below the power of our study, it is not of sufficient magnitude to warrant significant weighting for the selection of individual patients for an operation. Future prospective studies in this area are required, including rigorous preoperative and postoperative clinical and radiographic evaluation.

\section{CONCLUSIONS}

Normal-pressure hydrocephalus, a communicating form of adult hydrocephalus, is associated with the classic triad of gait, cognitive, and urinary disturbances in elderly patients. The DESH findings of tight high-convexity and medial subarachnoid spaces and enlarged Sylvian fissures with ventriculomegaly can be used for the diagnosis of NPH but may only encompass a portion of shuntresponsive adult hydrocephalus. The current study demonstrated urinary and dementia symptom improvement in both the presence and absence of DESH following shunting, with no significant differences between groups. Thus, shunt insertion for patients with adult hydrocephalus should not rely solely on the presence of preoperative DESH findings.

Disclosures: Abhay Moghekar-UNRELATED: Board Membership: I serve on the medical advisory board of the Hydrocephalus Association. It is an unpaid position; Travel/Accommodations/Meeting Expenses Unrelated to Activities Listed: Hydrocephalus Association, Comments: travel to the annual meeting advisory board meeting of the Hydrocephalus Association. Nafi Aygun-UNRELATED: Royalties: Elsevier, Cambridge University Press; Payment for Development of Educational Presentations: American College of Radiology. Ari M. Blitz-RELATED: Support for Travel to Meetings for the Study or Other Purposes: International Society for Hydrocephalus and CSF Disorders, Comments: partial reimbursement for travel for an educational talk on a related subject; UNRELATED: Grants/Grants Pending: National Institutes of Health, Comments: coinvestigator of FAIN U01DC013778 and R21 NS096497, prior grant support via the Principal Investigator Dr Daniele Rigamonti from Aesculab*; Payment for Lectures Including Service on Speakers Bureaus: French-Israeli Radiology course, Comments: partial reimbursement for travel for educational talks. *Money paid to the institution.

\section{REFERENCES}

1. Adams RD, Fisher CM, Hakim S, et al. Symptomatic occult hydrocephalus with "normal" cerebrospinal-fluid pressure: a treatable syndrome. N Engl J Med 1965;273:117-26 CrossRef Medline

2. Hashimoto M, Ishikawa M, Mori E, et al; Study of INPH on neurologic improvement (SINPHONI). Diagnosis of idiopathic normal pressure hydrocephalus is supported by MRI-based scheme: a prospective cohort study. Cerebrospinal Fluid Res 2010;7:18 CrossRef Medline

3. Marmarou A, Bergsneider M, Klinge $\mathrm{P}$, et al. The value of supplemental prognostic tests for the preoperative assessment of idiopathic normal-pressure hydrocephalus. Neurosurgery 2005;57 (3 Suppl):S17-28; discussion ii-v Medline

4. Relkin N, Marmarou A, Klinge P, et al. Diagnosing idiopathic normal-pressure hydrocephalus. Neurosurgery 2005;57(3 Suppl):S416; discussion ii-v Medline

5. Keong NC, Pena A, Price SJ, et al. Imaging of normal pressure hydrocephalus: theories, techniques, and challenges. Neurosurg Focus 2016;41:E11 CrossRef Medline

6. Mori E, Ishikawa M, Kato T, et al; Japanese Society of Normal Pressure Hydrocephalus. Guidelines for management of idiopathic normal pressure hydrocephalus: second edition. Neurol Med Chir (Tokyo) 2012;52:775-809 CrossRef Medline

7. Mendes GAS, de Oliveira MF, Pinto FCG. The Timed Up and Go Test as a diagnostic criterion in normal pressure hydrocephalus. World Neurosurg 2017;105:456-61 CrossRef Medline

8. Gallagher RM, Marquez J, Osmotherly P. Cognitive and upper limb symptom changs from a tap test in Idiopathic Normal Pressure Hydrocephalus. Clin Neurol Neurosurg 2018;10:92-96.

9. Kitagaki H, Mori E, Ishii K, et al. CSF spaces in idiopathic normal pressure hydrocephalus: morphology and volumetry. AJNR Am J Neuroradiol 1998;19:1277-84 Medline

10. Matsumae M, Kikinis R, Mórocz IA, et al. Age-related changes in intracranial compartment volumes in normal adults assessed by magnetic resonance imaging. J Neurosurg 1996;84:982-91 CrossRef Medline

11. Gammal TE, Allen MB, Brooks BS, et al. MR evaluation of hydrocephalus. AJR Am J Roentgenol 1987;149:807-13 CrossRef Medline

12. Narita $W$, Nishio $Y$, Baba $T$, et al. High-convexity tightness predicts the shunt response in idiopathic normal pressure hydrocephalus. AJNR Am J Neuroradiol 2016;37:1831-37 CrossRef Medline

13. Mostafa T, Craven C, Patel NA, et al. DESH negative normal pressure hydrocephalus: can patients still benefit from shunt insertion? Fluids and Barriers of the CN 2015;12(Suppl 1):O52 CrossRef 